
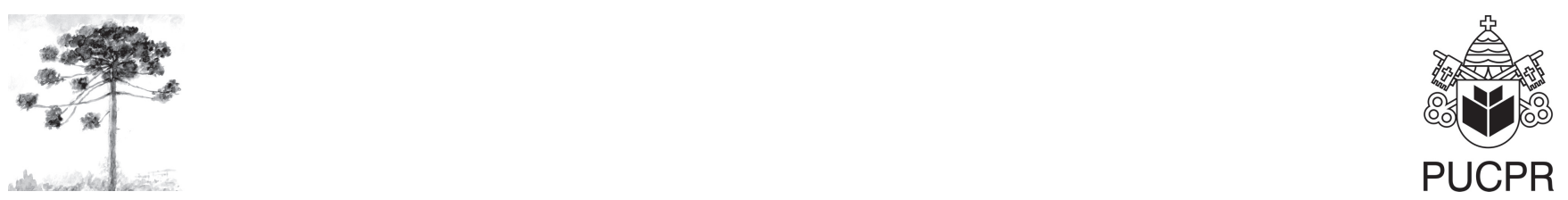

\title{
ESTUDO DAS INTERAÇÕES ENTRE INSETOS E Senecio brasiliensis Less. (ASTERACEAE) EM ÁREA EXPERIMENTAL NO CÂMPUS DA PONTIFÍCIA UNIVERSIDADE CATÓLICA DO PARANÁ, TOLEDO, BRASIL
}

\author{
Study of the interactions between insects and Senecio brasiliensis Less. \\ (ASTERACEAE) in experimental area in the Campus of the \\ Pontifical Catholic University of Parana, Toledo, Brazil
}

Marciele Solera ${ }^{1}$, Sonia Marisa Hefler ${ }^{2}$, Maria Cristina Zborowski de Paula ${ }^{3}$

${ }^{1}$ Acadêmica do Curso de Biologia da PUCPR e Bolsista do PIBIC, Curitiba, PR - Brasil, e-mail: marcissol@yahoo.com.br.

2 Orientadora do Projeto e docente da PUCPR - Câmpus Toledo. Toledo, PR - Brasil, e-mail: Sonia.hefler@pucpr.br

${ }^{3}$ Docente da PUCPR - Câmpus Toledo, Toledo, PR - Brasil, e-mail: Maria.zborowski@pucpr.br

\begin{abstract}
Resumo
Neste trabalho, foram observados e identificados os insetos visitantes e os eventos de interação em Senecio brasiliensis Less., levando em conta fatores ambientais. Foram analisadas seis parcelas ( $2 \mathrm{~m} \times 2 \mathrm{~m})$, em área da PUCPR Câmpus Toledo, em três horários diferentes. Registrou-se 204 insetos pertencentes a cinco ordens e 27 gêneros, observados em maior número no horário das $8 \mathrm{~h}$ às $10 \mathrm{~h}$ e especialmente entre as máximas de $25^{\circ} \mathrm{C}$ e $28^{\circ} \mathrm{C}$. Hymenoptera contribuiu com o maior número de indivíduos $(84 \%)$ e Hemiptera com o maior número de gêneros (10). Verificaram-se cinco eventos de interação, sendo alimentação/polinização o mais freqüente, especialmente em Hymenoptera e Lepidoptera. Esta última teve preferência por períodos de temperaturas mais altas, enquanto em Coleoptera, Hemiptera e Diptera não houve variação significativa na taxa de visitas, quando relacionada à temperatura.
\end{abstract}

Palavras-chave: Senecio brasiliensis; Interações; Insetos; Toledo. 


\begin{abstract}
This work was carried out to observe and identify visitor insects and their interaction with Senecio brasiliensis Lees., taking in account environment factors. It was analyzed six sites of $2 \mathrm{~m} X 2 \mathrm{~m}$, located in the PUCPR-Campus of Toledo, Parana, Brazil, in three periods of the day. It was registered 204 insects, related to five orders and 27 genera. Most of them was observed from 8:00 am to 10:00 am, specially when the highest temperature was 25 and $28^{\circ} \mathrm{C}$. Hymenoptera had the most of the individuals (84\%) and Hemiptera, most of the genera (10) identified. It was recorded five interaction events; feeding/pollination were the most frequent, especially in Hymenoptera and Lepidoptera. The later one preferred high temperature periods, while Coleoptera, Hemiptera and Diptera showed no significant variation of visits, in relation to temperature.
\end{abstract}

Keywords: Senecio brasiliensis; Interaction; Insects; Toledo.

\section{INTRODUÇÃO}

O reino vegetal, dado às suas características, é o que responde primeiramente às indagações e solicitações do homem. Na medida em que os conhecimentos sobre as plantas foram surgindo e acumulando, àquelas com propriedades medicinais passaram a ser objeto de interesse (1).

Espécies da família Asteraceae têm largo emprego como alimento, ornamental, inseticida natural, na medicina e fabricação de fármacos, podem também causar danos à agricultura, quando a população de ruderais não é mantida sob controle (2).

Senecio brasiliensis Less. (Asteraceae), conhecida popularmente por maria-mole, nativa da América do Sul, apresenta expressiva representatividade nos campos e áreas de regeneração natural do município de Toledo.

Segundo Kissmann e Groth (3), esta espécie pode demonstrar importância econômica positiva ou negativa. Positiva, quando causa um bom efeito ornamental. Negativa, pois contém uma série de alcalóides, ésteres de aminoálcoois que têm efeito negativo sobre o organismo.

Segundo Edwards e Wratten (4), os polinizadores de plantas com flores mais amplamente distribuídas são as abelhas, vespas e borboletas, embora muitas flores sejam polinizadas por besouros e moscas, e nos trópicos seja comum a polinização de flores por morcegos e aves. Em cada caso, os detalhes da estrutura da flor são maravilhosamente adaptados ao método da polinização.

A observação do tipo de interação que ocorre entre a fauna entomológica e flora medicinal/ tóxica podem revelar eventos que venham a contribuir com o equilíbrio vital destes organismos e do homem, porém ainda é pouco estudada. A maioria dos estudos concentra-se em espécies de importância agronômica ou nativa, conforme se observa nos trabalhos de Grutzmacher et al. (5), Boiça et al. (6), Manente-Balestieri e Machado (7).

O objetivo do presente trabalho é determinar e verificar a flutuação populacional dos insetos de acordo com a influência de fatores ambientais e de estágios de desenvolvimento de Senecio brasiliensis Less. em diferentes horários do dia, assim como observar os eventos de interação de insetos com a planta e caracterizar morfologicamente a espécie.

\section{MÉTODO}

O presente estudo foi realizado em área, a priori mecanizada e atualmente em estágio inicial de sucessão natural, localizada próximo a um remanescente de Floresta Estacional Semidecidual alterado, no Câmpus da Pontifícia Universidade Católica do Paraná, município de Toledo/PR.

Foram observados e identificados em nível de ordem e/ou gênero os insetos visitantes e os eventos de interação em seis inflorescências e duas anteses de cada uma das seis parcelas analisadas $(2 \mathrm{~m}$ x $2 \mathrm{~m}$ ). O tempo de observação em cada inflorescência foi de aproximadamente cinco minutos, sendo estas realizadas em três horários diferentes.

As seis parcelas foram escolhidas de forma aleatória e dispostas em locais diferentes, para observar e comparar se a localização destas teria 
alguma influência de fatores externos na interação dos insetos com a planta. As parcelas foram dispostas da seguinte forma: Parcela 1) próximo à borda de um remanescente de Floresta Estacional Semidecidual; Parcela 2) próximo a uma área mecanizada em ambiente aberto e com pouco contato antrópico; Parcela 3) localizada na porção central da área de estudo, em ambiente mais fechado e isolado; Parcelas 4, 5 e 6) localizadas próximo à rodovia/estrada de acesso ao Câmpus, com maior influência antrópica e em ambiente mais aberto, porém as parcelas estavam distantes uma das outras.

Durante as observações, foram levantados os seguintes dados: 1) condições ambientais de acordo com dados do Simepar (8); 2) estágio de maturação (antese, floração); 3) coleta e identificação de insetos visitantes, no laboratório de Zoologia da PUCPR Câmpus Toledo; 4) identificação dos eventos e duração dos eventos realizados pelo visitante: alimentação/polinização, reprodução, pouso, movimento e parado.

Para fins de análise de dados, foram considerados os insetos em nível de ordem, uma vez que nem todos os gêneros de insetos observados no campo foram coletados, sendo possível a identificação em nível genérico, apenas dos exemplares coletados.

\section{RESULTADOS}

Foram registradas visitas de 204 insetos, pertencentes a cinco ordens. Destas, a ordem que mais contribuiu com o número de exemplares foi Hymenoptera (84 indivíduos), e a que menos contribuiu (7 indivíduos) foi Diptera (Gráfico1).

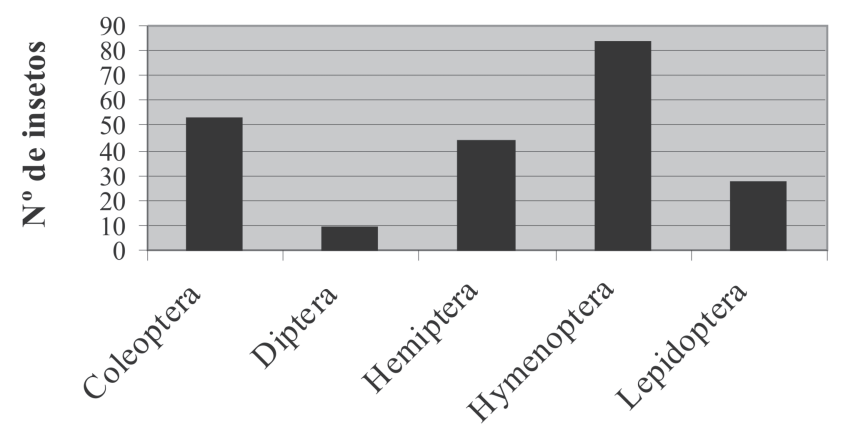

Nome da ordem

GRÁFICO 1 - Indicação da relação do número de insetos observados na área de estudo com a ordem a que pertencem
Foram classificados 27 gêneros com base na análise de 81 insetos coletados. Destes, a ordem que mais contribuiu com números de gêneros foi Hemiptera $(9,10)$ e a que menos contribuiu foi Hymenoptera (1) (Tabela 1).

TABELA 1 - Classificação dos visitantes florais de Senecio brasiliensis em área experimental no Câmpus da Pontifícia Universidade Católica do Paraná, Toledo, PR

\begin{tabular}{|c|c|}
\hline Ordem & Gênero \\
\hline Coleoptera & $\begin{array}{l}\text { Quedius } \\
\text { Calosoma } \\
\text { Diabrotica } \\
\text { Dasytes } \\
\text { Curculio } \\
\text { Cerotoma } \\
\text { Lagria } \\
\text { Porthmidius }\end{array}$ \\
\hline Diptera & $\begin{array}{l}\text { Chrysops } \\
\text { Liriomyza } \\
\text { Syrphus }\end{array}$ \\
\hline Hemiptera & $\begin{array}{l}\text { Lygocoris } \\
\text { Oncopeltus } \\
\text { Leptocoris } \\
\text { Dysdercus } \\
\text { Euryophthalmus } \\
\text { Oxycarenus } \\
\text { Anthocoris } \\
\text { Leptopterna } \\
\text { Lygaeus } \\
\text { Piesma }\end{array}$ \\
\hline Hymenoptera & Apis \\
\hline Lepidoptera & $\begin{array}{l}\text { Vanessa } \\
\text { Diatraeae } \\
\text { Elasmopalpus } \\
\text { Phoebis } \\
\text { Hypothyris }\end{array}$ \\
\hline
\end{tabular}

Das seis parcelas observadas, a parcela dois teve o maior número de insetos visitantes (62), sendo a maioria da ordem Hymenoptera. E o menor número de visitantes (11) ocorreu na parcela um.

Durante as observações, foram registrados cinco tipos de eventos realizados pelos insetos durante as visitas às inflorescências 
de Senecio brasiliensis: 1) alimentação/polinização, nestes os insetos estavam se alimentando e ao mesmo tempo podendo também estar polinizando; 2) pouso, o inseto pousava e permanecia na planta por poucos segundos; 3) reprodução, quando insetos estavam copulando; 4) parado, quando o inseto estava imóvel; 5) em movimento, quando o inseto andava pela inflorescência. Os dois últimos, considerados como eventos, mas não sendo possível a constatação da interação dos insetos com a planta durante as observações.

De todos os eventos, o de alimentação/ polinização foi o mais freqüente, realizado especialmente pela ordem Hymenoptera e Lepidoptera (Gráfico 2). Já os eventos de reprodução e "parado" foram os menos freqüentes, observados especialmente em Coleoptera e Hemiptera (Gráfico 2). O evento movimento é realizado mais comumente pela ordem Hemiptera.

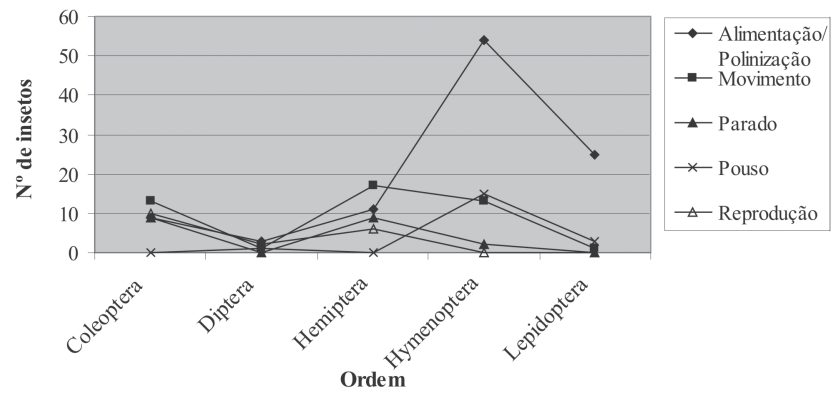

GRÁFICO 2 - Indicação dos eventos realizados pelas ordens dos insetos e o número de vezes em que foram observados

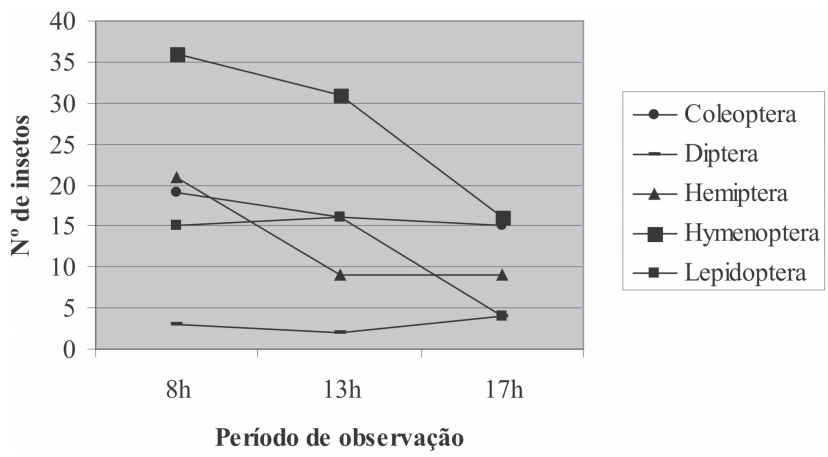

GRÁFICO 3 - Indicação do número de insetos observados em cada um dos três períodos de observação
O maior registro de insetos ocorreu no primeiro horário de observação, das $8 \mathrm{~h}$ às $10 \mathrm{~h}$ (Figura 3). Nesse horário, os insetos das ordens Coleoptera, Hemiptera e Hymenoptera foram observados em maior número. Já na ordem Lepidoptera, o maior número de indivíduos foi observado no segundo horário de observação, das 13 h às 15 h (Gráfico 3). Enquanto em Diptera, o maior número de insetos foi observado no terceiro horário, das $17 \mathrm{~h}$ às $19 \mathrm{~h}$ (Gráfico 3).

O maior número de insetos visitantes nas plantas amostradas ocorreu entre as máximas de $25^{\circ} \mathrm{C}$ e $28^{\circ} \mathrm{C}$, sendo esta a faixa de temperatura considerada ideal para visita, especialmente de Hymenoptera. Lepidoptera teve preferência por períodos de temperaturas mais altas, enquanto em Coleóptera, Hemiptera e Diptera não houve variação significativa na taxa de visitas, quando relacionada à temperatura (Gráfico 4).

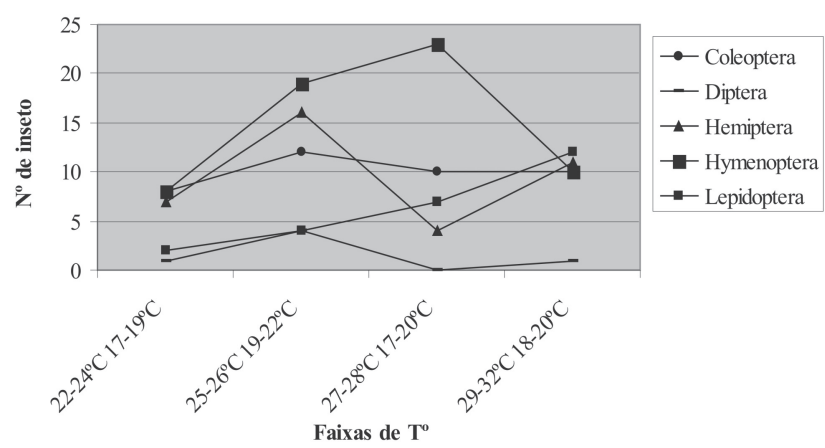

GRÁFICO 4 - Indicação do número de insetos em diferentes faixas de temperatura

\section{DISCUSSÕES E CONCLUSÕES}

O maior número de insetos visitantes ocorreu na parcela dois, em função desta se encontrar em fase de floração intensa desde o início das análises e por estar localizada próximo a uma área mecanizada em ambiente aberto e com pouco contato antrópico. Já a parcela um teve o menor número de visitantes, pois sua floração intensa ocorreu mais tardiamente do que as demais parcelas, não proporcionando condições para atração dos insetos.

A presença de insetos visitantes nas inflorescências foi mais observada em $S$. brasiliensis quando esta se encontrava em fase de floração, por apresentar flores vistosas, coloridas 
(amarela). Na fase de antese, o número de insetos visitantes foi menor, o que indica a preferência dos insetos em visitar a planta na fase de floração para a sua alimentação.

O evento alimentação/polinização realizado com maior freqüência pela ordem Hymenoptera e a partir do primeiro horário do dia corrobora com estudos realizados por Lenzi et al. (9) onde as abelhas iniciam a busca do néctar e pólen em flores de aroeira-vermelha, e Borba e Braga (14), onde ao longo de todo o período da manhã foi observada intensa atividade de indivíduos de Bombus atratus (Hymenoptera) visitando flores de Cuphea sp. (Lythraceae), nas quais coletavam néctar.

Já em estudos realizados por Taura e Laroca (15), em flores de Vassobia breviflora (Sendt.) Hunz. (Solanaceae) nas primeiras horas do período da manhã, constatou-se a freqüência reduzida de visitas de abelhas nestas flores.

De acordo com Manente-Balestieri e Machado (6), em estudo realizado sobre a Entomofauna visitante das flores de Cássia spectabilis (L.) DC. Leguminosae, os principais insetos polinizadores de vegetais são Hymenoptera (Apoidea). Isso foi comprovado neste trabalho, onde esta ordem contribuiu com o maior número de indivíduos, tendo como principal evento o de alimentação/polinização.

Para Ricklefs (10), as estruturas das flores e dos frutos limitam a diversidade de animais que executam esta função para uma espécie particular de planta. Por se tratar de uma espécie anemocórica, Senecio brasiliensis, quando em fase de senescência, favorece a dispersão dos aquênios pelo vento (11), provavelmente isso fez com que o número e a diversidade de insetos visitantes não fossem tão altos, uma vez que não necessita destes para sua dispersão. Outro fator que pode ter favorecido para a baixa diversidade é área de estudo, que por estar em fase de sucessão e ser bastante alterada, provavelmente, muitas espécies pioneiras ainda não apresentem atrativos para determinados grupos de insetos.

Segundo Antonini et al. (12), Lepidoptera forrageia ocorre preferencialmente em luminosidade e temperatura mais altas, concordando com este estudo onde representantes desta ordem tiveram maior ocorrência em temperaturas altas e dias mais ensolarados. Verificou-se que em dias nublados, houve pouca interação de todas as ordens de insetos analisadas no presente estudo. Fatores abióticos influenciam bastante as taxas de visitas, pois a combinação de altos valores de luminosidade e temperatura favorece as visitas. Porém outros fatores (ventos) podem estar influenciando na interação dos insetos com a planta. No entanto, se as condições abióticas não forem adequadas às limitações fisiológicas de cada grupo taxonômico, a abundância de visitantes será afetada (12).

Segundo Karam et al. (11), a maior incidência das fases vegetativas de Senecio ocorre num período de maior precipitação, enquanto a floração e frutificação restringem-se a períodos menos chuvosos e de temperaturas mais elevadas. Com base em dados do Simepar (8), esta incidência foi observada, sendo que a fase de floração e frutificação mais intensa de $S$. brasiliensis ocorreu em períodos em que houve baixa precipitação e índices de temperaturas mais elevadas, e a fase de brotação ou antese ocorreu em períodos de maior precipitação.

Santana et al. (13) lembram que é importante conhecer o horário de visitação do inseto a uma flor para determinar a sua importância na polinização, pois para ser um polinizador efetivo é necessário que este visite as flores, normalmente quando há disponibilidade de pólen, e no horário de receptividade de seus estigmas. Neste estudo, constatou que as abelhas (Apis sp.) preferem visitar a flor para a busca de pólen em temperaturas mais elevadas e em alta luminosidade. Nas primeiras horas do período da manhã, possivelmente a temperatura encontra-se mais baixa e a umidade mais elevada, complementado por redução de néctar e pólen das flores (15).

A presença de insetos permanentes ou transitórios foi uma observação constante no trabalho realizado por Karam et al. (11), insetos broqueadores de caule e de botões florais, mas especialmente desfolhadores como Phaedon confinis, lagartas de Pericopis sacrifica e Phaedon sp. e o besouro Systena s-littera tenuis, foram encontrados isolados ou associados, principalmente em Senecio brasiliensis e S. selloi. Estas espécies não foram identificadas no presente estudo durante o período de observações.

Verificou-se que a diversidade de insetos visitantes em Senecio brasiliensis foi abaixo do esperado quando comparado com outros 
trabalhos já realizados por outros autores em outras áreas. Porém teve a presença das principais ordens entomológicas.

Por outro lado, o maior número de visitas foi da ordem Hymenoptera (Apis sp.), com o evento alimentação/polinização, confirmando as propriedades melíferas desta planta, mencionadas por (3).

Observou-se que a temperatura influencia no número e na diversidade de algumas ordens de insetos visitantes, como Lepidoptera, que teve maior incidência em períodos de temperaturas mais elevadas $\left(29-32^{\circ} \mathrm{C}\right)$, enquanto Hymenoptera em períodos de temperatura intermediária $\left(27^{\circ}-28^{\circ} \mathrm{C}\right)$. Para Antonini et al. (12) os lepidópteros também são muito susceptíveis as variações de clima, sendo necessário para o vôo condições ideais que incluam altas taxas de luminosidade e altas temperaturas além da ausência de ventos fortes, pois em geral as borboletas têm estrutura corpórea delicada e sensível. Já as abelhas sociais (Apidae) forragearam desde o início do dia até o final da tarde, mostrando alta tolerância principalmente a variações na temperatura e luminosidade.

Verificou-se ainda que o horário das $8 \mathrm{~h}$ às $10 \mathrm{~h}$ é o mais indicado para observações de insetos em Senecio brasiliensis.

Entre as cinco ordens, aquela que ocorreu em maior número foi Hymenoptera, o que está relacionado à capacidade melífera da planta, já mencionada anteriormente.

\section{AGRADECIMENTOS}

Aos acadêmicos Adriano Banaki, pela colaboração na identificação dos insetos, e Luciane Kerber da Silva, pela ajuda nas observações e coletas de dados em campo. Ao Simepar, pelo fornecimento dos dados de temperatura. Ao PIBIC, pela concessão da Bolsa de Iniciação Científica, permitindo a realização deste trabalho.

\section{REFERÊNCIAS}

1. Rizzo JA, Monteiro MSR, Bitencourt C. Utilização de Plantas Medicinais em Goiânia. Congresso Nacional de Botânica, 36, Curitiba, 1985, Anais Brasília, IBAMA. 1990;(2):692.
2. Ribeiro JELS, Hopkins MJG, Vicentini A, Sothers CA, Costa MAS, Brito JM, et al. Flora da Reserva Ducke: Guia de Identificação das plantas vasculares de uma floresta de terra-firme na Amazônia Central. Manaus: INPA;1999. 648 p.

3. Kissmann GK, Groth D. Plantas Infestantes e Nocivas. 2 ed. São Paulo: BASF; 1999. 404 p.

4. Edwards PJ, Wratten SD. Ecologia das interações entre insetos e plantas. São Paulo: EPU; 1981. 1 p.

5. Grützmacher AD, Nakano O, Martins JFS, Loeck AE, Grützmacher DD. Consumo foliar de cultivares de arroz irrigado por Spodoptera frugifera (J.E. Smith) (Lepidoptera: Noctuidae). An Soc Entomol. 1999;28(3):519-525.

6. Manente-Balestieri LDCF, Machado LLV. Ecologia, comportamento e Bionomia, An. Soc Entomol Brasil. 1999;28(3): 429-437.

7. Boiça Jr. AL, Santos TM, PASSILONGO, J. Trigona spinipes (Fabr.) (Hymenoptera: Apidae) em espécies de maracujazeiro: flutuação populacional, horário de visitação e danos às flores. Neotropical Entomology. 2004;33(2):135-139.

8. Instituto Tecnológico Simepar - SIMEPAR. [cited 2005 set 4]. Disponível em: www.simepar.br.

9. Lenzi M, Orth AI, Laroca S. Associação das abelhas silvestres (Hym., Apoidea) visitantes das flores de Schinus terebinthifolius (Anacardiaceae), na Ilha de Santa Catarina (sul do Brasil). Acta Biol Par. 2003;32(1, 2, 3, 4):107-127.

10. Ricklefs RE. A economia da natureza: um livro-texto em ecologia básica. 3. ed. Rio de Janeiro: Guanabara Koogan; 1996.

11. Karam FSC et al. Fenologia de quatro espécies tóxicas de Senecio (Asteraceae) na região Sul do Rio Grande do Sul. Pesq. Vet. 2002;36.22(1):33-39. 
12. Antonini et al. Diversidade e comportamento dos insetos visitantes florais de Stachytarpheta glabra Cham. (Verbenaceae), em uma área de Campo Ferruginoso. Outro Preto, Mg. Neotropical Entomology. 2005; 34(4):555-564.

13. Santana MP; Carvalho CF; Souza B, Morgado LN. Abelhas (Hymenoptera: Apoidea) visitantes das flores do feijoeiro, Phaseolus vulgaris L., em Lavras e Ijaci-MG. Ciênc. Agrovet. 2002;26(6):1119-1127.

14. Borba LE, Braga SIP. Biologia reprodutiva de Psendolaelia corcovadensis (Orchidaceae): melitofilia e autocompatibilidade em uma Laeliinae basal. Revista Brasil Bot. 2003; 26(4):541-549.

15. Taura HM, Laroca S. Biologia da Polinização: interações entre as abelhas (Hym., Apoidea) e as flores de Vassobia breviflora (Solanaceae). Acta Biol Par. 2004; 33(1,2,3,4):143-162.

Recebido: 01/06/2006

Received: 06/01/2006

Aceito: $03 / 10 / 2006$

Accepted: 10/03/2006 